\title{
Seminal Fructose Concentration in Man Infertility and the Fructose Test's Meaning In Diagnosis Reason of Azoospermia Man
}

\author{
Nguyen Thi Trang ${ }^{1 *}$, Vu Thi Huyen ${ }^{2}$, Nguyen Thi Thuy Linh ${ }^{3}$ and Trieu Tien Sang ${ }^{4}$ \\ ${ }^{1}$ Department of Biomedical and Genetic, Hanoi Medical University, Vietnam \\ ${ }^{2}$ Hanoi Medical University, Vietnam \\ ${ }^{3}$ Faculty of Biotechnology, VNU University of Science, Vietnam \\ ${ }^{4}$ Military Medical University, Vietnam
}

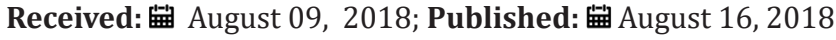

*Corresponding author: Nguyen Thi Trang, Department of Biomedical and Genetic, Hanoi Medical University, Vietnam

\begin{abstract}
Background: This is a review from 2 studies that determine the concentration of seminal fructose and show the correlations between seminal fructose concentration with sperm concentration, vitality and motility (progressive). Besides, these studies also show the reason of man infertility cases which azoospermia and no seminal fructose. Materials and methods: Include random 60 normozoospermia, 60 oligozoospermia semen. Determine fructose concentration by ROE method. 25 azoospermia cases with no fructose had been examined and proceed percutaneous epididymis sperm aspiration (PESA) to find the infertility reason.
\end{abstract}

Results: Fructose concentration of normozoospermia: 1,601 $\pm 0,604(\mathrm{~g} / \mathrm{l})$ significant lower than oligozoospermia: 1,881 $\pm 0,640$ (g/l). Fructose seminal concentration has negative correlations with sperm concentration $(R=-0.156 ; p>0.05)$; sperm vitality $(R=-0.065 ; p>0.05)$; sperm progressive motility $(\mathrm{R}=-0.186 ; \mathrm{p}<0.05)$. Examine and process percutaneous epididymis sperm aspiration (PESA), 25 azoospermia with no fructose cases have diagnosed congenital bilateral absence of the vas deferens (CBAVD).

Conclusions: Seminal fructose concentration of normozoospermia group is significant lower than oligozoospermia group. Fructose seminal concentration has negative correlations with sperm concentration, concentration and motility. $100 \%$ azoospermia cases with no seminal fructose have diagnosed CBAVD.

Keywords: Infertility; Seminal Fructose; Azoospermia; CBAVD

Abbreviations: CBAVD: Congenital Bilateral Absence Of The Vas Deferens; PESA: Percutaneous Epididymis Sperm Aspiration; HCl: Hydrochloric Acid

\section{Introduction}

WHO 2010 divided man infertility into 3 groups: normozoospermia (sperm concentration $>=15$ billion $/ \mathrm{ml}$ ), oligozoospermia (sperm concentration $<15$ billion/ml) and azoospermia (no sperm) [1]. There are many studies determined seminal fructose and its correlations with concentration, vitality and motility of sperm. Azoospermia is the reason in 20\% infertility man [2] and the common reason of azoospermia is CBAVD [3], [4]. The purpose of the studies is using seminal fructose test to replace percutaneous epididymis sperm aspiration (PESA) to diagnosis CBAVD. Fructose is essential for spermatozoa metabolism and spermatozoa motility [5]. Fructose is an energy source for spermatozoa. It is produced by the seminal vesicles with some contribution from the ampulla of the ductus deferens $[6,7]$ Determination of seminal fructose concentration has been used in examination of obstructive azoospermia and inflammation of male accessory glands $[8,9]$. The role of fructose concentrations in seminal plasma for total and sperm density has been investigated by several authors. Rajalakhshmi M et al. [10] and Gonzales GF [11] reported that an increase in sperm concentration is often accompanied by a decrease in fructose concentration in seminal plasma, because sperm using fructose as the primary source of energy [10,11]. However, others studies have also shown that fructose concentrations in seminal plasma of patients with oligozoospermia and azoospermia did not decrease as compared to normal men.

\section{Materials and Methods}

The studies select semen of infertility men (age 18-50) visited in Centre of genetics counselling of Hanoi medical university hospital from 3/2017 - 5/2018. After analyzing the semen, results of sperm concentration, vitality and motility were selected. Random choose 60 normozoospermia cases and 60 oligozoospermia cases to determine seminal fructose concentration by ROE improved method [12]. Fructose content in seminal plasma was determined 
by the resorcinol method where fructose reacts with resorcinol in concentrated hydrochloric acid $(\mathrm{HCl})$ solution to form a red compound. Measure the coloric complex of Zinc and Fructose at a wavelength of $560 \mathrm{~nm}$ against blanks. Besides, 25 patients that azoospermia with no fructose had been examined and proceed percutaneous epididymis sperm aspiration (PESA) to find the infertility reason in Andrology Department, Hanoi Medical University Hospital. Use SPSS version 16.0 to analyze the results.

\section{Results and Discussion}

Abdella MA [13] have reported that seminal fructose concentration is higher in oliogozoospermia and lower in azoospermia and asthenospermia [13]. Fructose concentration in norrmozoopermia is significant lower than oligozooseprmia [14]. Table 1 and Figures 1-3 shows that seminal fructose in oligozoospermia is significant higher than oliogozoospermia $(p<0,05)$. Besides, other semen characteristics include sperm concentration, vitality and progressive motility in oligozoospermia are significant lower than normozoospermia $(\mathrm{p}<0,001)$. That means when seminal fructose decreases, sperm concentration, vitality and progressive motility increase. In some cases that sperm count and sperm concentration are too, high, seminal fructose may decrease lower than normal. To clear these correlations between seminal fructose and other semen characteristics, the studies analyze selected results by Pearson's correlation. The results show the negative correlations between seminal fructose and sperm concentration $(R=-0,156$ and $p>0,05)$, sperm vitality ( $R$ $=-0,065$ and $p>0,05)$ and sperm progressive motility $(R=-0,186$ and $\mathrm{p}<0,05)$. Gonzales GF [15], Orakwe JC [16] and Mahmoud HH [17] have reported similar conclusions. Fructose in semen is the source of energy of every sperm activities. The higher of sperm concentration, vitality and motility asked for more energy, so fructose is lower [18,19]. The study of Lu (2007) reported when sperm motility increased, fructose decreased and in vitro, sperm continued using fructose [18].

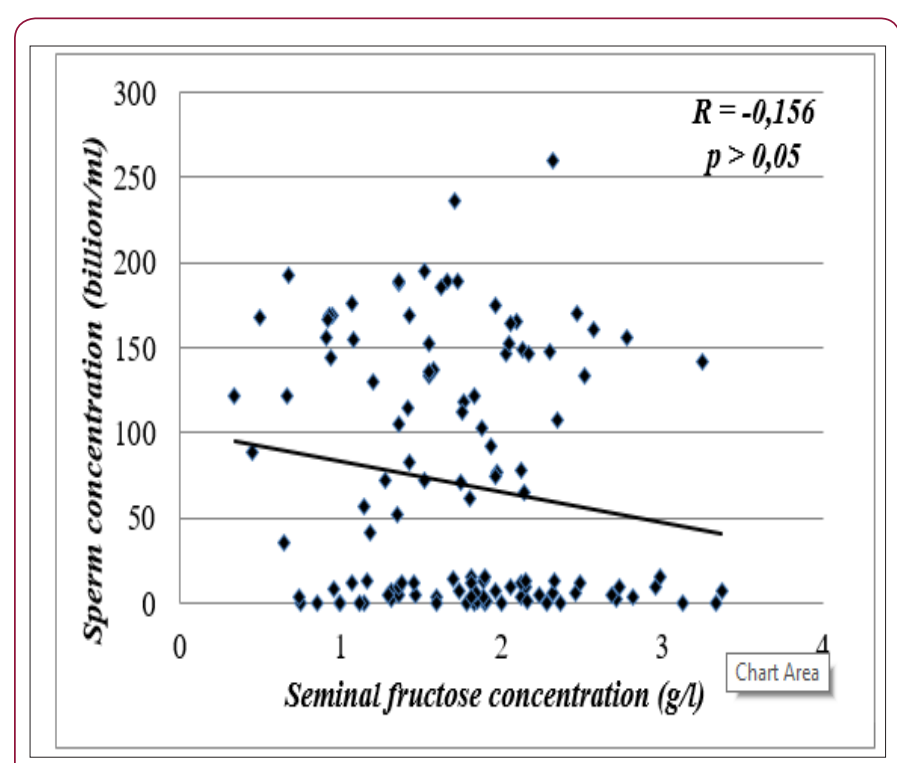

Figure 1.

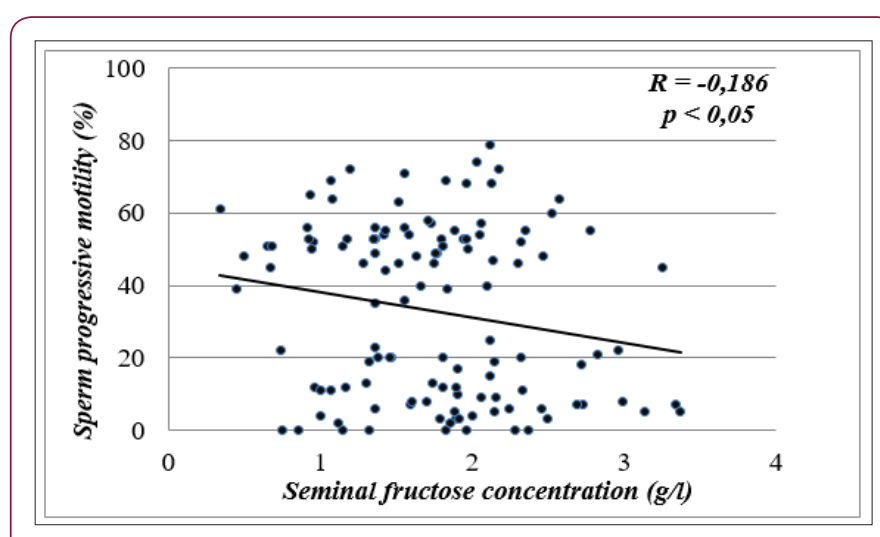

Figure 2.

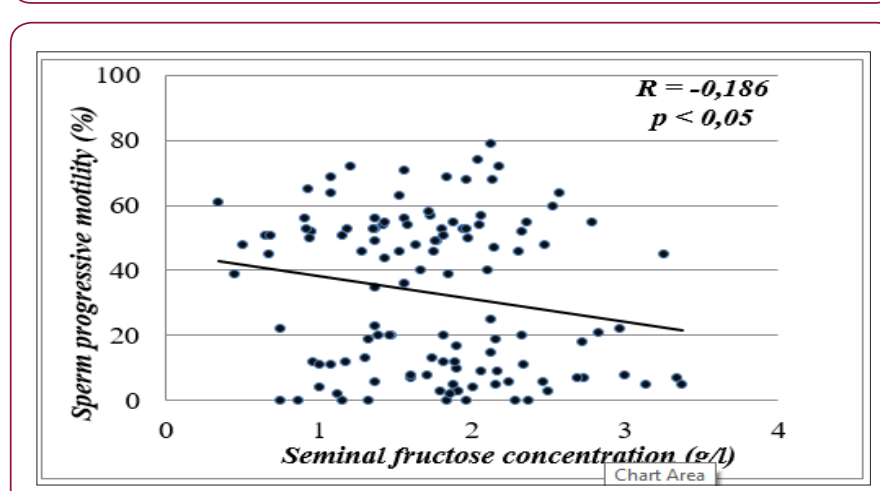

Figure 3.

Table 1: Seminal fructose and some characteristics of the semen in 2 groups.

\begin{tabular}{|c|c|c|c|}
\hline Group & $\begin{array}{c}\text { Normozoospermia } \\
\mathbf{( N = 6 0 )}\end{array}$ & $\begin{array}{c}\text { Oligozoospermia } \\
\mathbf{( N = 6 0 )}\end{array}$ & $\mathbf{P}$ \\
\hline Variables & $1,601 \pm 0,604$ & $1,881 \pm 0,640$ & $<0,05$ \\
\hline $\begin{array}{c}\text { Fructose } \\
\text { concentration } \\
\text { (billion/ml) }\end{array}$ & $133,808 \pm 48,215$ & $5,633 \pm 4,992$ & $<0,001$ \\
\hline Vitality (\%) & $86,483 \pm 3,218$ & $58,183 \pm 18,114$ & $<0,001$ \\
\hline $\begin{array}{c}\text { Progessive } \\
\text { motility (\%) }\end{array}$ & $54,667 \pm 9,278$ & $11,250 \pm 10,157$ & $<0,001$ \\
\hline Fructose & $1,601 \pm 0,604$ & $1,881 \pm 0,640$ & $<0,05$ \\
\hline
\end{tabular}

Normal seminal fructose concentration confirms the role of testosterone and the function of vesicles and vas deferens are normal [20]. The absence of both sperm and fructose correlates with the obstruction in CBAVD or retrograde ejaculation [1,21]. Especially, the correlation between azoospermia and fructose in CBAVD had been proved by many authors [22]. All 25 cases azoospermia with no seminal fructose have been examined and proceed percutaneous epididymis sperm aspiration (PESA) by andrologies to find the infertility reason. The result shows that the reason in all the cases is CBAVD. Fructose concentration in obstructive azoospermia cases is lower than normal or zero [23]. By other side, non-obstructive azoospermia, fructose concentration 
usually higher or equal than normal [21]. Inflammation the reproductive glands causes temporary obstruction, so that sperm count and seminal fructose concentration may decrease, but rarely absence both of them [21,24].

Follow to WHO 2010 [1], Gonzales G F [15], Kumar R [4], some characteristics of the semen in obstructive infertility include:
a. Azoospermia.
b. Seminal fructose low or zero.
c. Volume of semen $<1,5 \mathrm{ml}$.
d. $\quad \mathrm{pH}$ of semen $<7$.

Our studies show that all 25 azoospermia cases with no seminal fructose are CBAVD. Because of that reason, seminal fructose test is considered to replace epididymis sperm aspiration (PESA) to find the infertility reason. Fructose is a main carbohydrate source in seminal plasma and necessary for sperm motion [25,26]. The measurement of seminal fructose has been used in most laboratories. Therefore, the World Health Organization manual recommends measurement of seminal fructose as a marker of seminal vesicular function [27]. Methods for determination of seminal fructose mainly include gas chromatography, indole coloration, and resorcinol coloration. In particular, the resorcinol method has been used widely in clinical andrology laboratories for its simplicity of operation, high specificity, and no need for special instrument. Fructose in semen is the source of energy for all sperm activities. The higher of sperm concentration, and vitality and motility asked for more energy, so fructose is lower [7,28]. Normal seminal fructose concentration confirms the role of testosterone and the function of vesicles and vas deferens are normal [29].

In this study, negative correlations were observed between seminal fructose and sperm concentration $(R=-0,156$ và $p>0,05)$, sperm vitality $(R=-0,065$ và $p>0,05)$ and sperm progressive motility $(R=-0,186$ và $p<0,05)$. This finding is in line with that of Gonzales GF [11], Orakwe JC [30] and Mahmoud HH [31]. Fructose in semen is the source of energy of every sperm activities. The higher of sperm concentration, vitality and motility asked for more energy, so fructose is lower [28,32]. Lu et al. [28] reported seminal fructose concentration decreased, sperm concentration and mobility increased [28].

Lewis Jones et al. [29] found that fructose concentrations were inversely ratio to sperm motility with $R=-0,062(p<0.05)$ [29]. However, Andrade Rocha FT [33] confirmed that seminal fructose concentration was related to sperm concentration, survival, motility and morphology, but the results were not statistically significant [33]. In the study of Amidu N [34], seminal fructose concentration was negatively correlated with sperm motility $(R=-0.04)$ but not statistically significant [34]. Fructose concentrations were inversely ratio to sperm concentration $(R=-0.21)$ with correlation was significant at 0.05 level [35]. Fructose is the major glycolysable substrate of seminal plasma and is widely accepted as a marker of seminal vesicle function [34-36]. Inflammation may lead to atrophy of the seminal vesicles and low seminal fructose concentration.
When ejaculatory ducts are blocked, fructose concentration in seminal plasma usually decreases and may become undetectable $[34,37]$. Additionally, seminal plasma fructose concentration determination is useful for auxiliary diagnosis of obstructive and nonobstructive azoospermia. Seminal fructose concentration in non-obstructive azoospermia is usually higher than or equal to that in males of normal fertility [33]. However, fructose concentration in seminal plasma of patients with obstructive azoospermia is usually absent or significantly lower than that in men of normal fertility $[34,36]$. Absence of seminal fructose has also been found in patients with congenital vas deferens-seminal vesicle developmental defect [39]. Therefore, our results are consistent with most of the results of studies in the world.

Normal seminal fructose concentration confirms the role of testosterone and the function of vesicles and vas deferens are normal [36]. The absence of both sperm and fructose correlates with the obstruction in CBAVD or retrograde ejaculation $[9,13]$. Especially, the correlation between azoospermia and fructose in CBAVD had been proved by many authors [5]. In this study, all 25 cases azoospermia without seminal fructose have been examined and proceed percutaneous epididymal sperm aspiration (PESA) by andrologist to find the infertility reason. The result shows that the reason in all the cases is CBAVD. Fructose concentration in obstructive azoospermia cases is lower than normal or absent [9]. By other side, In human with non-obtructive azoopermia, fructose concentration usually higher or equal than normal [9]. Inflammation the reproductive glands causes temporary obstruction, so that sperm count and seminal fructose concentration may decrease, but rarely absence both of them $[9,40,41]$.

\section{Conclusion}

Seminal fructose concentration of normozoospermia group is significant lower than oligozoospermia group. Fructose seminal concentration has negative correlations with sperm concentration, concentration and motility. $100 \%$ azoospermia cases with no seminal fructose are congenital bilateral absence of the vas deferens - CBAVD.

\section{References}

1. WHO (2010) Laboratory manual for the examination and processing of human semen ( $5^{\text {th }}$ Edn.). Cambridge University Press, Cambridge, United Kingdom.

2. American Urological Association, American Society for Reproductive Medicine (2006) The male infertility Best Practice Policy Committee of the American Urological Association, Practice Committee of the American Society for Reproductive Medicine. Report on optimal evaluation of the infertile male. Fertility and Sterility 86(5): 202-209.

3. Rajalakhshmi M, Sherma RS, David GFX, Kapur MM (1989) Seminal fructose in normal and infertile men. Contraception 39(3): 299-306.

4. Biswas S, Ferguson KM, Stedronska J, Baffoe G, Mansfield MD, et al. (1978) Fructose and hormone levels in semen: their correlations with sperm counts and motility. Fertility and Sterility 30(2): 200-204.

5. Videla E, Blanco AM, Galli ME, Fernández Collazo E (1981) Human seminal biochemistry: fructose, ascorbic acid, citric acid, acid phosphatase and their relationship with sperm count. Andrologia 13(3): 212-214. 
6. Schoenfeld C, Amelar RD, Dubin L, Numeroff M (1979) Prolactin, fructose, and zinc levels found in human seminal plasma. Fertil Steril 32(2): 206-208.

7. Biswas S, Ferguson KM, Stedronska J, Baffoe G, Mansfield MD, et al. (1978) Fructose and hormone levels in semen: their correlations with sperm counts and motility. Fertility and Sterility 30(2): 200-204.

8. Carpino A, De Sanctis V, Siciliano L, Maggiolini M, Vivacqua A, et al. (1997) Epididymal and sex accessory gland secretions in transfusiondependent beta-thalassemic patients: evidence of an impaired prostatic function. Exp Clin Endocrinol Diabetes 105(3): 169-174.

9. Manivannan B, Bhande SS, Panneerdoss S, Sriram S, Lohiya NK (2005) Safety evaluation of long-term vas occlusion with styrene maleic anhydride and its non-invasive reversal on accessory reproductive organs in langurs. Asian J Androl 7(2): 195-204.

10. Rajalakhshmi M, Sherma RS, David GFX, Kapur MM (1989) Seminal fructose in normal and infertile men. Contraception 39(3): 299-306.

11. Gonzales GF (2001) Function of seminal vesicles and their role on male fertility. Asian J Androl 3(4): 251-258.

12. Roe JH (1934) A colorimetric method for the determination of fructose in blood and urine. J Biol Chem 107: 15-22.

13. Abdella MA, Omer EA, Badruldeen H Al Aabed (2010) Biochemical markers in semen and their correlation with fertility hormones and semen quality among Sudanese infertile patients. African Journal of Biochemistry Research 4(11): 255-260.

14. Amidu N, Owiredu WKBA, Bekoe MAT, L Quaye (2012) The impact of seminal zinc and fructose concentration on human sperm characteristic. Journal of Medical and Biomedical Sciences 1(1): 14-20.

15. Gonzales GF (2001) Function of seminal vesicles and their role on male fertility. Asian J Andrology 3 (4): 251-258.

16. Orakwe JC, Chukwuezi FO, Ebu GU (2010) True corrected seminal fructose in male infertility Nigerians a preliminary study. Nigerian Journal of Clinical Practice 13(1): 84-86.

17. Mahmoud HH et al (2014) The key role of Zinc in enhancement of total antioxidant levels in spermatozoa of patients with Asthenozoospermia. Reproductive Biology and Endocrinology 12: 3-8.

18. Lu CJ, Fang Chen, Hui Ru Xu, Yu Feng Huang, Nian Qing Lu (2007) Standardization and quality control for determination of fructose in seminal plasma. Journal of Andrology 28(2): 207-213.

19. Biswas S, Ferguson KM, Stedronska J, Baffoe G, Mansfield MD, et al. (1978) Fructose and hormone levels in semen: their correlations with sperm counts and motility. Fertility and Sterility 30(2): 200-204.

20. WHO (2010). Laboratory manual for the examination and processing of human semen ( $5^{\text {th }}$ Edn.). Cambridge University Press, Cambridge, United Kingdom.

21. Buckett WM, Lewis Jones DI (2002) Fructose concentrations in seminal plasma from men with non - obstructive azoospermia. Arch Andrology 48(1): 23-27.

22. Lipshultz LI, Howards SS (1991) Infertility in the Male. In: Lipshultz LI, Howards SS (Eds) (2 $2^{\text {nd }}$ Edn.). Mosby - Year Book Inc, Missouri, United States: 133-135.

23. Manivannan B, Bhande SS, Panneerdoss S, S Sriram, NK Lohiya (2005) Safety evaluation of long - term vas occlusion with styrene maleic anhydride and its non-invasive reversal on accessory reproductive organs in langurs. Asian J Andrology 7(2): 195-204.
24. Dohle GR (2003) Inflammatory associated obstructions of the male reproductive tract. Andrologia 35 (5): 321-324

25. Johnsen O, Eliasson R (1987) Evaluation of a commercially available kit for the colorimetric determination of zinc in human seminal plasma. Int J Androl 10(2): 435-440.

26. Roe JH (1934) A colorimetric method for the determination of fructose in blood and urine. J Biol Chem 107: 15-22.

27. Franken DR, Oehninger S (2012) Semen analysis and sperm function testing. Asian J Androl 14(1): 6-13.

28. Lu CJ, Fang Chen, Hui Ru Xu, Yu Feng Huang, Nian Qing Lu (2007) Standardization and quality control for determination of fructose in seminal plasma. Journal of Andrology 28(2): 207-213.

29. Buckett WM, Lewis Jones DI (2002) Fructose concentrations in seminal plasma from men with non - obstructive azoospermia. Arch Andrology 48(1): 23-27.

30. Orakwe JC, Chukwuezi FO, Ebu GU (2010) True corrected seminal fructose in male infertility Nigerians a preliminary study. Nigerian Journal of Clinical Practice 13(1): 84-86.

31. Mahmoud HH (2014) The key role of Zinc in enhancement of total antioxidant levels in spermatozoa of patients with Asthenozoospermia. Reproductive Biology and Endocrinology 12: 3-8.

32. Biswas S, Ferguson KM, Stedronska J, Baffoe G, Mansfield MD, et al (1978) Fructose and hormone levels in semen: their correlations with sperm counts and motility. Fertility and Sterility 30(2): 200-204.

33. Andrade Rocha FT (2001) Sperm parameters in men with suspected infertility. Sperm characteristics, strict criteria sperm morphology analysis and hypoosmotic swelling test. J Reprod Med 46(6): 577-582.

34. Ndovi TT, Choi L, Caffo B, Parsons T, Baker S, et al. (2006) Quantitative assessment of seminal vesicle and prostate drug concentrations by use of a noninvasive method. Clin Pharmacol Ther 80(2): 146-158.

35. Raj V, Vijayan AN, Joseph K (2013) Naked eye detection of infertility using fructose blue-a novel gold nanoparticle based fructose sensor. Biosens Bioelectron 54: 171-174.

36. WHO (2010). Laboratory manual for the examination and processing of human semen ( $5^{\text {th }}$ Edn.). Cambridge University Press, Cambridge, United Kingdom.

37. Coppens L (1997) Diagnosis and treatment of obstructive seminal vesicle pathology. Acta Urol Belg 65(2): 11-19.

38. Buckett WM, Lewis Jones DI (2002) Fructose concentrations in seminal plasma from men with non - obstructive azoospermia. Arch Andrology 48(1): 23-27.

39. Kise H, Nishioka J, Satoh K, Okuno T, Kawamura J, et al. (2000) Measurement of protein $C$ inhibitor in seminal plasma is useful for detecting agenesis of seminal vesicles or the vas deferens. J Androl 21(2): 207-212.

40. Dohle GR (2003) Inflammatory associated obstructions of the male reproductive tract. Andrologia 35(5): 321-324.

41. Yassa DA, Idriss WK, Atassi ME, SK Rao (2001) The diagnostic value of seminal $\alpha$-glucosidase enzyme index for sperm motility and fertilizing capacity. Saudi Medical Journal 22 (11): 987-991. 
ISSN: 2574-1241

DOI: 10.26717/BJSTR.2018.08.001598

Nguyen Thi Trang. Biomed J Sci \& Tech Res

(c) (P) This work is licensed under Creative BY Commons Attribution 4.0 License

Submission Link: https://biomedres.us/submit-manuscript.php

$\begin{array}{ll}\text { BIOMEDICAL } & \text { Assets of Publishing with us } \\ \text { RESEARCHES } & \text { Global archiving of articles } \\ & \text { - Immediate, unrestricted online access } \\ & \text { - Rigorous Peer Review Process } \\ & \text { - Authors Retain Copyrights }\end{array}$

